\title{
DONACIÓN VOLUNTARIA DE SANGRE DE CORDÓN UMBILICAL EN EL HOSPITAL UNIVERSITARIO VIRGEN DE LA ARRIXACA (2010-2011). SITUACIÓN ACTUAL E INFLUENCIA DE LAS VARIABLES OBSTÉTRICO- FETALES
}

\section{DELIBERATE DONATION OF UMBILICAL CORD BLOOD IN THE UNIVERSITY HOSPITAL VIRGEN DE LA ARRIXACA (2010-2011). CURRENT SITUATION AND INFLUENCES OF THE OBSTETRICAL-FOETAL VARIABLES.}

\author{
Soto Herrero, Verónica * \\ Martínez Rojo, Carmen * \\ Morillas Guijarro, Daniel * \\ Rodríguez Portilla, Natalia * \\ Martínez, Román, Concepción *
}

E-mail contacto: vesohe33@regmurcia.com

* Servicio de partos. Hospital Universitario Virgen de la Arrixaca. Murcia.

\section{RESUMEN}

DONACIÓN VOLUNTARIA DE SANGRE DE CORDÓN UMBILICAL EN EL HOSPITAL UNIVERSITARIO VIRGEN DE LA ARRIXACA (2010-2011). SITUACIÓN ACTUAL E INFLUENCIA DE LAS VARIABLES OBSTÉTRICO- FETALES 
Objetivos: A principios de 2010 el Hospital Universitario Virgen de la Arrixaca puso en marcha, en el servicio de partos, el protocolo de donación voluntaria de sangre de cordón umbilical (SCU). Desde la reflexión y la autoevaluación, planteamos un análisis de nuestra situación actual a través del estudio de la influencia de las variables obstétrico-fetales en la calidad de las muestras.

Métodos: Con este fin hemos planteado un trabajo de tipo observacional, descriptivo, retrospectivo y de corte transversal desde mayo de 2010 a noviembre de 2011, con el objetivo de aumentar la calidad de las unidades de SCU y optar por una gestión eficiente que haga sostenible el proyecto.

Resultados: Se obtuvieron 123 donaciones potenciales de sangre de cordón y del análisis multivariable de las mismas obtuvimos una correlación positiva significativa entre el número de leucocitos, las semanas de gestación y el parto vaginal. El peso inicial de la unidad se incrementó significativamente a mayor peso del recién nacido y paridad de la donante.

Conclusiones: Como conclusiones del estudio nos planteamos esbozar posibles factores predictivos que permitan seleccionar las muestras de mayor calidad y sean complemento de los actuales estándares elaborados por el Banco Público de Málaga. En este sentido nuestros resultados sugieren que en los partos con más semanas de gestación, paridad de la gestante y peso del recién nacido, así como en los partos vaginales, encontramos mayores posibilidades de obtener muestras de alta calidad.

Palabras claves: Matrona, sangre de cordón umbilical, selección de donantes.

\section{ABSTRACT}

DELIBERATE DONATION OF UMBILICAL CORD BLOOD IN THE UNIVERSITY HOSPITAL VIRGEN DE LA ARRIXACA (2010-2011). CURRENT SITUATION AND INFLUENCES OF THE OBSTETRICAL-FOETAL VARIABLES.

Aims: At the beginning of 2010, the University Hospital la Arrixaca, set up the protocol, in the birth delivery service, of umbilical cord blood (U.C.B.) donation. From the reflexion and the self-evaluation, we set out an analysis of 
our current situation through the study of the influences of the obstetricalfoetal variables in the samples quality.

Method: On this purpose, we set out an observational descriptive, retrospective and cross-section study from May 2010 to November 2011, so as to increase the quality of the UCB units and to opt for efficient management that would make the project sustainable.

Results: We obtained 123 potential donations of umbilical cord blood and out of the multivariable analysis we obtained a positive and significant correlation between the number of leukocytes, the weeks of pregnancy and the vaginal birth. The initial weight of the unit significantly increased to a heavier weight of the new born and the amount of times a donor has given birth.

Conclusions: As conclusions from this study we suggest to outline possible predictive factors that would allow us to select the best quality samples and would be a complement to the current standards produced by the Public Bank of Malaga. In this sense our results suggest that in the deliveries with more weeks of pregnancy, in the amount of times a woman has given birth and in the weight of the new born as well as in the vaginal deliveries we can find more possibilities of obtaining high quality samples.

Key Words: Midwife, umbilical cord blood, donors' selection.

\section{INTRODUCCIÓN}

A principios de 2010, el Hospital Universitario Virgen de la Arrixaca (H.U.V.A.) fue acreditado como centro extractor de Sangre de Cordón Umbilical (S.C.U) iniciándose el proceso de recogida y envío de donaciones voluntarias.

La puesta en marcha del protocolo de recogida, envío y transporte al banco público de sangre de Málaga, del que dependemos, ha sido y es una labor compleja que ha precisado de formación, motivación, colaboración interprofesional y entre distintos niveles asistenciales. Son estas ideas de mejora en la gestión de las muestras las que, como matronas impulsoras y partícipes en esta puesta en marcha de la recogida de SCU, nos hacen 
plantearnos una reflexión desde los paradigmas de la calidad en salud atendiendo a esa satisfacción de las pacientes, los profesionales que colaboran, la implicación de las direcciones de enfermería y el uso de herramientas para la mejora continua ${ }^{1}$. Desde esta mirada, elaboramos un proyecto de evaluación, análisis y propuestas de mejora del protocolo de recogida y envío de SCU del que presentamos en este trabajo una primera parte del mismo; el análisis de nuestra situación actual y estudio de la influencia de las variables obstétricofetales en la calidad de las muestras finales obtenidas. Entendemos que la unidad de sangre de cordón es de calidad cuando logra superar los criterios mínimos establecidos por el Banco de sangre en cuanto a peso inicial de la unidad y número de leucocitos, y por tanto de células nucleadas totales.

Pero ¿por qué este interés en el almacenamiento de SCU? ¿Cuál es la utilidad real de estos bancos públicos de almacenamiento de SCU? En la actualidad los transplantes hematopoyéticos se clasifican según el donante y la fuente de células progenitoras. En el caso de estos últimos, encontramos una fuente celular adecuada en la médula ósea, en sangre periférica y en SCU${ }^{2}$. La SCU contiene células madre multipotenciales dentro de las cuales se encuentran las células madre hematopoyéticas, capaces de dividirse y crear más células madre y transformarse en leucocitos hematíes o plaquetas ${ }^{3}$.

Entre las ventajas de este tipo de transplantes de SCU encontramos la ausencia de riesgo en donantes, está al alcance de minoría étnicas, presenta escasa trasmisión de ciertas enfermedades, es de fácil almacenamiento y disponibilidad, muestra menor aloreactividad y es de bajo coste y elevado potencial hematopoyético ${ }^{4}$. Los inconvenientes son mínimos pero es una realidad que las muestras poseen una menor celularidad hematopoyética lo que limita su uso en adultos además de presentar la posibilidad de transmisión de enfermedades congénitas no detectables con los actuales test.

Según el Plan Nacional de Sangre de Cordón Umbilical ${ }^{5}$ pueden ser 
donantes voluntarias aquellas mujeres sanas que no tengan riesgo de transmitir enfermedades infecciosas ni genéticas, expresen su deseo de ser donantes y den a luz en una maternidad autorizada para realizar extracciones. Las donantes deberán firmar un consentimiento informado y no presentar ninguno de los criterios de exclusión expuestos en el Plan Nacional.

Tradicionalmente la SCU era considerada un producto de desecho sanitario $^{6}$ hasta que en 1988 un equipo del hospital Saint Louis dirigido por la Dra. Elian Gluckman realiza con éxito el primer transplante de SCU para el tratamiento de la anemia de Fanconi ${ }^{7}$. Tras estos primeros logros se conciben estructuras organizativas en torno a la idea de conservar y usar la $\mathrm{SCU}^{8}$. El primer banco de SCU se creó en Nueva York en 1992. En España los bancos de sangre de cordón pueden ser públicos, privados y mixtos, actualmente existen 7 bancos públicos autonómicos de almacenamiento de SCU. Así mismo, son más de 160 las maternidades acreditadas para la extracción de $\mathrm{SCU}^{9}$. Cada banco público pone a disposición del Registro Español de Donantes de Médula Ósea (REDMO) sus datos y del registro internacional NETCORD. Los organismos internacionales, determinan unos estándares generales de recogida y procesamiento aunque cada banco establece sus propios criterios de aptitud $^{3,7}$. En nuestro hospital las extracciones se realizan siguiendo el procedimiento proporcionado por el Banco de SCU de Málaga donde se establecen como criterios de valoración inicial de las unidades antes de su envío, el peso de la bolsa mayor de $110 \mathrm{gr}$ y un recuento de leucocitos comprendido entre $5 \times 10^{6} / \mathrm{mL}$ y $22 \times 10^{6} / \mathrm{mL}$ y de manera indirecta mediante el calculo aproximado de las células nucleadas totales $(\mathrm{CNT})>12 \times 10^{8}$.

El punto guía teórico para la extracción y conservación de SCU, lo establece el Plan Nacional de Sangre de Cordón Umbilical, aprobado por la Comisión de Transplantes del Consejo Interterritorial en marzo de 2008 y que ha contribuido a aumentar la calidad de las unidades almacenadas que en la actualidad son unas 45.000 de las 60.000 que se plantearon como meta. 
Previamente, aparece en España el RD $1301 / 2006^{10}$ que regula legislativamente la donación pública de la $\mathrm{SCU}^{9}$. Es una satisfacción comprobar que España es el segundo país en número absoluto de unidades almacenadas del mundo 5 .

De este modo, las matronas queremos contribuir con nuestro trabajo y nuestras investigaciones a consolidar y mejorar el futuro de los transplantes hematopoyéticos, otorgando a este proyecto de donación de SCU la importancia social que merece e intuimos que la clave de la excelencia del mismo, es la adecuada preselección para una recogida de muestras de calidad suprema con representación de minorías étnicas que nos permitan a través de una gestión eficiente la sostenibilidad de este proyecto de almacén de vidas.

\section{OBJETIVO GENERAL}

Aumentar la calidad de las unidades de sangre de cordón recogidas en el H.U.V.A.

\section{OBJETIVOS ESPECÍfICOS}

1. Describir la situación actual del procedimiento de recogida voluntaria de SCU en el paritorio del H.U.V.A.

2. Determinar la influencia de las variables obstétrico-fetales en el número de leucocitos de la muestra y peso inicial de la unidad de sangre de cordón en gramos de las muestras donadas en el paritorio del H.U.V.A.

3. Determinar la influencia de las variables turno y profesional responsable del procedimiento de recogida de sangre de cordón, en el número de leucocitos y peso inicial en gramos de la unidad de sangre de cordón.

\section{MÉTODOS Y PACIENTES (SUJETOS) MATERIAL Y MÉTODO}

La investigación se llevó a cabo en el servicio de partos del H.U.V.A., Murcia. 
El estudio fue de tipo observacional, descriptivo, retrospectivo y de corte transversal de los procedimientos de donación voluntaria de sangre de cordón umbilical realizados desde mayo de 2010 a noviembre de 2011 .

Se analizaron 123 donaciones potenciales de sangre de cordón en el paritorio para su procesamiento en el Banco de Sangre de Cordón de Málaga. Entendemos por donantes potenciales todas aquellas gestantes que han manifestado su deseo de donar SCU, han firmado el consentimiento y han sido incluidas en el registro de posibles donantes del servicio de partos.

\section{Variables}

1. Independientes

a. Semanas de Gestación.

b. Paridad.

c. Presentación fetal.

d. Tipo de parto.

e. Turno en que se realiza la recogida de la unidad de sangre de cordón.

f. Sexo del recién nacido.

g. Peso del recién nacido.

h. Profesional que interviene en la recogida de la unidad de sangre de cordón.

i. Iniciativa para la donación.

\section{Dependientes}

a. Recogida de la sangre de cordón.

b. Causas de no recogida.

c. Envío de la unidad de sangre de cordón.

d. Causas de no envío de la sangre de cordón.

e. Número de leucocitos de la muestra de sangre de cordón/ uL. 
f. Unidades procesadas en el Banco de Sangre de Cordón de Málaga.

g. Peso inicial de la unidad de sangre de cordón.

h. Causas del no procesamiento de la unidad de sangre de cordón.

Las características materno-fetales de las donantes potenciales fueron recogidas del libro de registro de partos y completadas con el registro informático SELENE de dicho servicio.

La iniciativa para la donación, la recogida, así como las causas de no recogida de sangre de cordón, el envío, las causas de no envío, y el número de leucocitos de la muestra, fueron obtenidas del registro de donaciones de sangre de cordón y la hoja de transporte de las unidades del cual dispone nuestro servicio.

El peso inicial de la unidad de sangre de cordón, el procesamiento y las causas de no procesamiento fueron obtenidas a través de la información aportada por el Banco de Sangre de Cordón Umbilical de Málaga.

Para el análisis estadístico se utilizó el paquete SPSS versión 15.0. Para la realización de gráficos y tablas además del SPSS también se utilizó el programa Microsoft Office Excel 2007.

De este modo para la descripción de las variables cuantitativas y cualitativas se empleó la media, mediana, mínimo, máximo, frecuencia absoluta y porcentaje según correspondía, estableciendo en todos los casos un Índice de Confianza del $95 \%$. Se utilizó para la comparación de medias de las variables con distribución normal la T de Student. Para establecer el grado de correlación entre las variable cuantitativas, se empleo el Índice de Correlación de Pearson. Se asume una significación estadística para valores de $p<0,05$.

En la estrategia de búsqueda se han consultado fuentes digitales tales 
como páginas webs de organismos oficiales sanitarios nacionales e internacionales y fundamentalmente bases de datos en ciencias de la salud como MEDLINE, Cuiden, Lilacs, Cochrane, Pubmed, Scielo y Teseo además de bases de datos en ciencias sociales del CSIC, Dialnet así como otras tesis doctorales digitalizadas a través de TDR.

Los criterios de búsqueda se han establecido en función de las bases de datos o recursos utilizados, empleando los términos matrona, donación, sangre de cordón umbilical, Banco de sangre de cordón y factores obstétricos.

\section{RESULTADOS:}

Desde mayo de 2010 hasta noviembre de 2011 se registraron 123 donaciones potenciales de SCU en nuestro servicio. Realizamos un análisis de las características obstétrico- fetales de las posibles donantes cuyo resultado puede verse en la tabla 1: Características Obstétrico-fetales de las posibles donantes (Variables cuantitativas) y en la tabla 2: Características Obstétricofetales de las posibles donantes (Variables cualitativas).

Estos partos fueron atendidos en el 47,2\% $(n=58)$ por matronas del servicio mientras que el 52,8\% $(n=65)$ fueron partos donde colaboraron en el proceso tanto el ginecólogo como la matrona. La recogida de sangre se realizó fundamentalmente durante el turno de día 62,6\% $(n=77)$. La iniciativa de donar sangre de cordón partió principalmente de la propia gestante $(64,2 \%)$. En el $22,8 \%$ de los casos fueron los profesionales los que ofertaron la posibilidad de realizar el procedimiento. Debemos señalar que existe un $13 \%$ de donaciones potenciales en las que no se registró de donde partió la iniciativa de la donación. De las 123 gestantes que manifestaron su deseo de donar, no se llegó a recoger en 8 casos (6,5\%) siendo la causa principal la falta de material para la extracción en el momento del parto. De las 115 unidades de sangre que sí llegaron a ser recogidas, 48 unidades fueron desechadas en el propio paritorio $(41,74 \%)$, no llegando a ser enviada al banco de sangre, principalmente porque la muestra tenía baja celularidad o porque la unidad no 
alcanzaba el peso mínimo requerido.

Desde el inicio del procedimiento se han enviado un total de 67 unidades

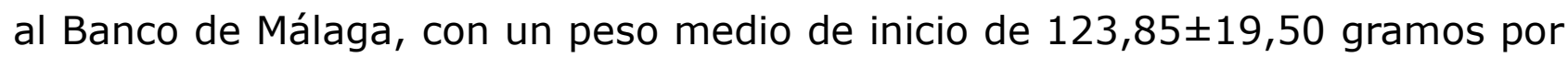
unidad (min= 86 gr. /máx.= 177 gr.) y un número de leucocitos medio de $13.73 \times 10^{3} / \mathrm{uL}$. De estas 67 unidades enviadas fueron procesadas 22 $(17,9 \%)$.

Hubo un punto de inflexión en abril de 2011, en el que además de considerar como criterio de exclusión para el envío de unidades al banco el peso inicial de la bolsa inferior a 110 gramos, la muestra debía tener un número determinado de leucocitos (y por tanto de CNT) para considerarla óptima. Hasta este momento sólo se procesaban un $20 \%$ de las muestras enviadas; Al introducir el cálculo de la celularidad de la muestra pasamos a aumentar el número de muestras procesadas hasta un 46,87\%. (Figura 1: Envío y procesamiento de las unidades antes y después del cálculo de la celularidad)).

Las causas de no procesamiento mas frecuentes en todos los casos han sido la baja celularidad de la muestra en primer lugar (18,7\%), en segundo lugar el bajo volumen de la unidad $(9,8 \%)$ y por último la presencia de coágulos en la muestra $(3,3 \%)$.

Con respecto al análisis de la posible relación entre las variables en las que se centró la investigación, obtuvimos los siguientes resultados; hayamos una correlación positiva directa entre la variable semanas de gestación y el número de leucocitos de la muestra (Coeficiente de Correlación =0,360) alcanzando la significación estadística $(p=0,009)$.

(Figura 2: Correlación entre semanas de gestación y nº de leucocitos/uL.)

Por el contrario, no se obtuvo una correlación significativa entre las semanas 
de gestación y el peso inicial de la unidad de sangre de cordón.

También existe una correlación positiva entre el peso del recién nacido y el peso inicial de la unidad de sangre de cordón (Coeficiente de Correlación= $0,375)$ alcanzando la significación estadística $(p=0,003)$.

(Figura 3: Correlación entre el peso del recién nacido y el peso inicial de la unidad de sangre de cordón en gramos.)

Al contrastar los leucocitos de la muestra con el tipo de parto, obtuvimos que la media de leucocitos en los partos vaginales había sido muy superior a la media de leucocitos en las cesáreas, alcanzando una diferencia estadísticamente significativa $(p=0,001)$.

(Tabla 3: Correlación entre el tipo de parto y el peso y el número de leucocitos de la unidad).

Sin embargo, con respecto al peso inicial de la unidad de sangre de cordón, aunque fue superior en los partos vaginales (124,31 gr frente a 119,67 gr en las cesáreas) la diferencia no logró la significación estadística.

No se encontró una diferencia estadísticamente significativa con respecto al número de leucocitos y peso inicial de la unidad, ni con el profesional que intervino en la recogida de la sangre de cordón, ni con el turno en el cual se realizó la técnica.

Cuando contrastamos el sexo del recién nacido con el número de leucocitos de la muestra, encontramos que las muestras obtenidas de varones tenían una media de leucocitos superior a la obtenida de mujeres (14.67 x $10^{3} / \mathrm{uL}$ frente a $12.76 \times 10^{3} / \mathrm{uL}$ ) que aunque no alcanzaba la significación estadística, se situaba muy próxima a ella $(p=0,069)$. La diferencia del peso medio inicial de las unidades de sangre de cordón obtenida de varones aunque fue superior a la obtenida en mujeres no se encuentra una diferencia 
significativa (124,76 gr en varones frente a $122,74 \mathrm{gr}$. en mujeres).

Al analizar el peso medio inicial de las unidades de sangre según la paridad obtuvimos que conforme aumentaba el numero de hijos, el peso de las unidades era mayor (Coeficiente de Correlación $=0,287$ ) alcanzando esta diferencia la significación estadística $(p=0,026)$ mientras que no se obtuvo relación entre la paridad y el número de leucocitos de la muestra.

(Figura 4: Peso inicial de la unidad de sangre de cordón según paridad de la donante.)

\section{DISCUSIÓN}

Una vez obtenidos nuestros resultados nos disponemos a compararlos con los obtenidos por otros investigadores. En primer lugar atendiendo a la relación entre semanas de gestación y número de leucocitos de la muestra, y por tanto de células nucleadas totales, podemos afirmar que a mayor semanas de gestación aumenta el número de CNT y por tanto aumenta la calidad de la muestra. Este hallazgo es compartido por numerosos autores ${ }^{8,11}$. Con respecto a la influencia del tipo de parto sobre el número de CNT de la unidad, podemos afirmar que es mayor en los partos vaginales frente a las cesáreas, dato que coincide con la investigación de Aroviita $^{12}$, pero que discrepa con los resultados obtenidos por otros autores ${ }^{8,11}$ que no han encontrado diferencia significativa entre ambos, aunque si han encontrado un mayor número de CNT en los partos instrumentados frente a los partos eutócicos.

Con respecto al volumen de la bolsa coincidimos con las investigaciones actuales $^{8,11,12}$ en que a mayor peso del recién nacido, mayor peso inicial de la unidad de sangre. Todos los estudios consultados coinciden en afirmar que el peso de la placenta es un buen predictor de las $\mathrm{CNT}^{8,11}$. En nuestro caso es un dato que no se registra y por tanto una limitación en nuestro estudio, en el que sólo disponemos de los pesos de los recién nacidos. 
Aunque en nuestros resultados observamos una correlación positiva entre la paridad y el peso en gramos de la bolsa, no hemos encontrado ningún autor que relacionara estas dos variables por lo que dejamos una línea abierta para futuras investigaciones.

Aunque el sexo del recién nacido solo rozó la significación estadística al relacionarlo con el número de leucocitos de la muestra, otros estudios establecen que los recién nacidos varones presentan una mayor cantidad de $\mathrm{CNT}^{8,12}$ frente a las mujeres.

No hemos encontrado ningún estudio que relacionara el turno de trabajo ni el profesional que realiza el procedimiento con la calidad de las unidades. Otros investigadores han estudiado la influencia de otros factores diferentes que pueden contribuir a mejorar la calidad como situar al recién nacido sobre el abdomen materno ${ }^{13}$ o la acidosis fetal ${ }^{14}$.

\section{CONCLUSIÓN}

Aunque en el paritorio del H.U.VA. seguimos el procedimiento del Banco de Málaga donde se establecen como criterios de inclusión gestaciones mayores o iguales a 34 semanas y peso del recién nacido iguales o superiores a $2500 \mathrm{gr}$, nuestros resultados nos sugieren que conforme aumentan las semanas de gestación y el peso del recién nacido, también aumentarán la calidad de las unidades.

Del mismo modo aunque la paridad y el tipo de parto no son criterios a considerar en el procedimiento, nuestros resultados sugieren que la multiparidad y los partos vaginales también son posibles factores predictivos de unidades de mayor calidad. 


\section{BIBLIOGRAFÍA}

(1) Chandia Vidal Y. Calidad en Salud. 2006.

(2) Trasplante de progenitores hemopoyéticos. Anales Sis. San Navarra: SciELO España; 2006.

(3) Alberich X. Conservación y aplicaciones terapéuticas de las células madre de cordón umbilical. Matronas Profesión 2009; 10(1):14-16.

(4) Torres Almagro MC, Bernal Gutiérrez MA, Corchete Marín Y. Estado actual de la donación de sangre de cordón umbilical en España. Evidentia 2010; $7(30)$.

(5) Organización Nacional de Transplantes. Plan Nacional de Sangre de Cordón Umbilical. Marzo 2008.

(6) Larios Risco D. Donación y uso privativo de la sangre del cordón umbilical: aspectos jurídicos. DS: Derecho y salud 2007; 15(2):181-216.

(7) Carreras E. Optimización en la recogida de sangre del cordón umbilical. Medicina clínica 2007; 129(15):574-575.

(8) Solves Alcaina P, Perales Marín A, Mirabet Lis A, Brik Spinellib M, Soler García, A y Roig Oltra, R. Selección de donantes y recogida de las unidades en un banco de sangre de cordón umbilical. Medicina Clínica 2007; 129(15):561.

(9) Carreras E. Preservación de la sangre de cordón umbilical. An. Pediat. Contin. 2010; 8(6):329-33.

(10) Ministerio de Sanidad y Consumo. Real Decreto 1301/2006, de 10 de noviembre, por el que se establecen las normas de calidad y seguridad para la donación, la obtención, la evaluación, el procesamiento, la preservación, el almacenamiento y la distribución de células y tejidos humanos y se aprueban las normas de coordinación y funcionamiento para su uso en humanos. B.O.E num.270. Sábado 11 de Noviembre de 2006.

(11) Jones J, Stevens CE, Rubinstein P, Robertazzi RR, Kerr A, Cabbad MF. Obstetric predictors of placental/umbilical cord blood volume for transplantation. Obstet Gynecol 2003; 188(2):503-509.

(12) Aroviita P, Teramo K, Hiilesmaa V, Kekomäki R. Cord blood hematopoietic progenitor cell concentration and infant sex. Transfusion 2005; 45(4):613-621.

(13) Grisaru D, Deutsch V, Pick M, Fait G, Lessing JB, Dollberg S, et al. Placing the newborn on the maternal abdomen after delivery increases the volume and CD34 cell content in the umbilical cord blood collected: an old maneuver with new applications. Obstet. Gynecol. 1999; 180(5):1240-1243.

(14) Juutistenaho S, Eskola M, Sainio S, Aranko K, Kekomäki R. Association of stress-related perinatal factors and cord blood unit hematopoietic progenitors is dependent on delivery mode. Transfusion 2010;50(3):663. 


\section{TABLAS}

Tabla 1: Características Obstétrico- fetales de las posibles donantes (Variables cuantitativas)

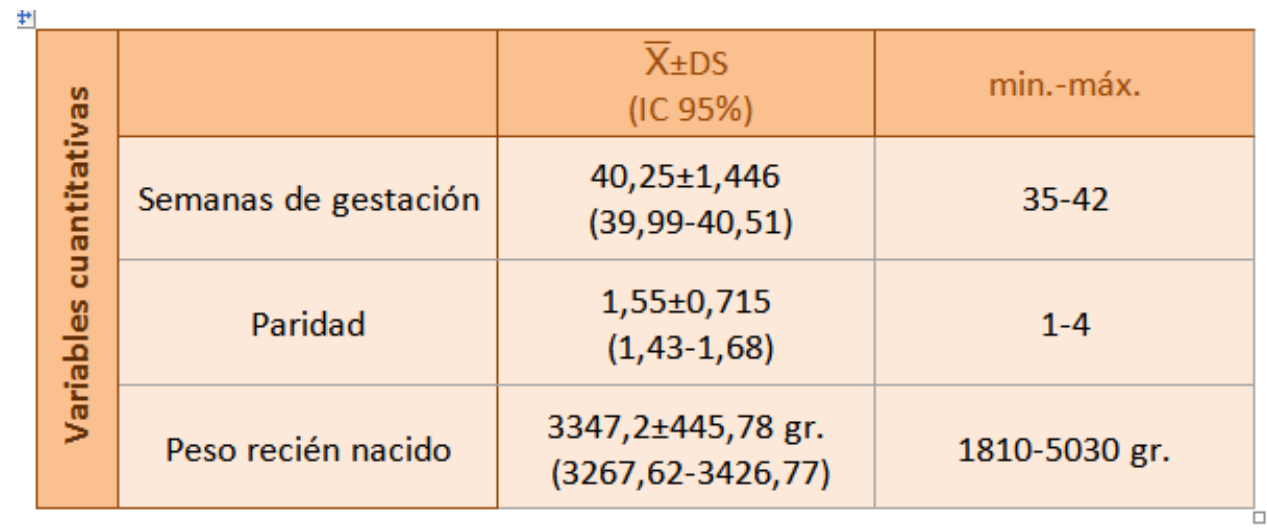

Tabla 2: Características Obstétrico- fetales de las posibles donantes (Variables cualitativas)

\begin{tabular}{|c|c|c|c|c|}
\hline & & & Frecuencia absoluta & $\%$ \\
\hline \multirow{4}{*}{ 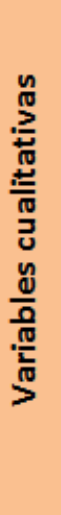 } & Tipo de parto & $\begin{array}{l}\text { Vaginal } \\
\text { Cesárea }\end{array}$ & $\begin{array}{l}102 \\
21\end{array}$ & $\begin{array}{l}82,90 \% \\
17,10 \%\end{array}$ \\
\hline & Presentación & $\begin{array}{c}\text { Cefálica } \\
\text { Otros }\end{array}$ & $\begin{array}{c}114 \\
8\end{array}$ & $\begin{array}{r}92,70 \% \\
6,50 \%\end{array}$ \\
\hline & \multirow[t]{2}{*}{ Sexo recién nacido } & Mujer & 57 & $46,30 \%$ \\
\hline & & Varón & 66 & $53,70 \%$ \\
\hline
\end{tabular}

Tabla 3: Correlación entre el tipo de parto y el peso y el número de leucocitos de la unidad.

\begin{tabular}{|c|ccc|}
\hline \multicolumn{2}{|c|}{} & $\begin{array}{c}\overline{\mathbf{x}} \text { Leucocitos } \\
(\mathbf{p}=0,001)\end{array}$ & $\begin{array}{c}\overline{\mathbf{x}} \text { Peso inicial } \\
\text { de la unidad } \\
(\mathbf{p}>0,05)\end{array}$ \\
\hline \multirow{2}{*}{ Tipo de parto } & Vaginal & $14,57 \times 10^{3} / \mathrm{UI}$ & $124,31 \mathrm{gr}$ \\
& Cesárea & $10,29 \times 10^{3} / \mathrm{uL}$ & $119,67 \mathrm{gr}$ \\
\hline
\end{tabular}




\section{FIGURAS:}

Figura 1: Envío y procesamiento de las unidades antes y después del cálculo de la celularidad.

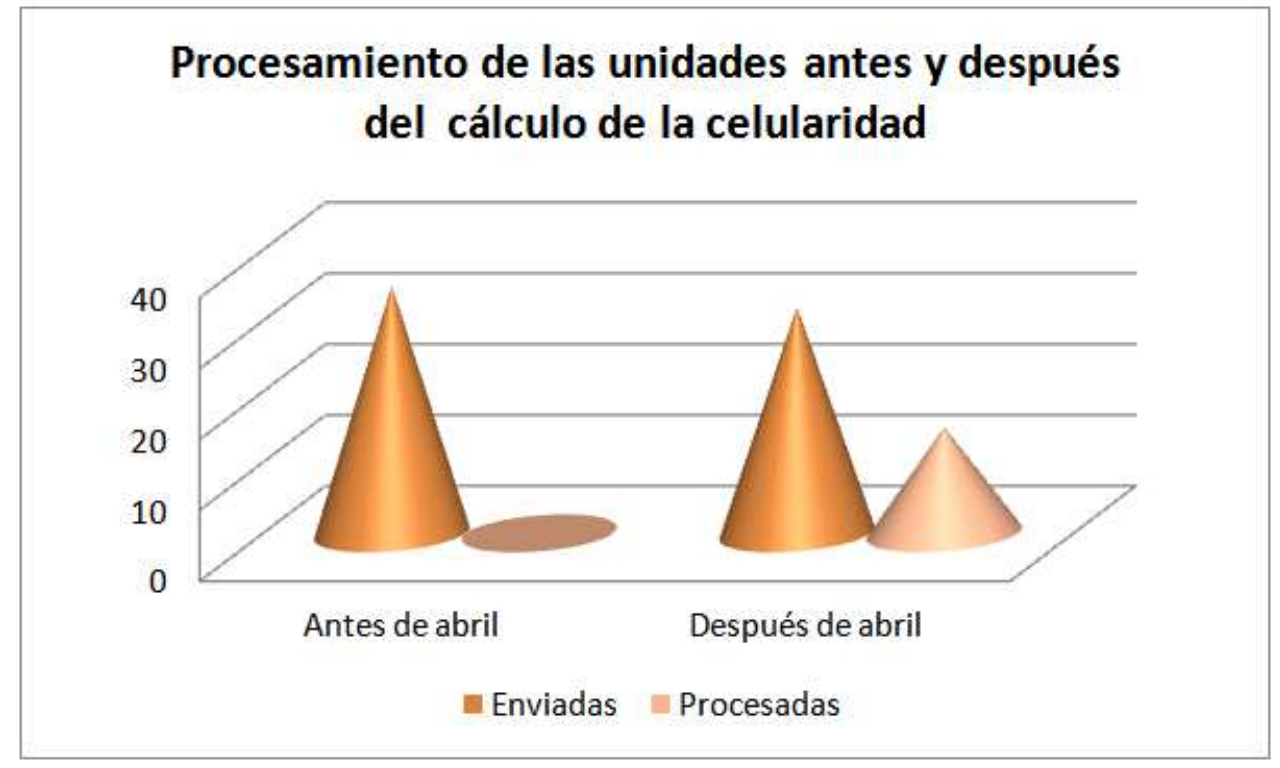

Figura 2: Correlación entre semanas de gestación y número de Leucocitos uL.

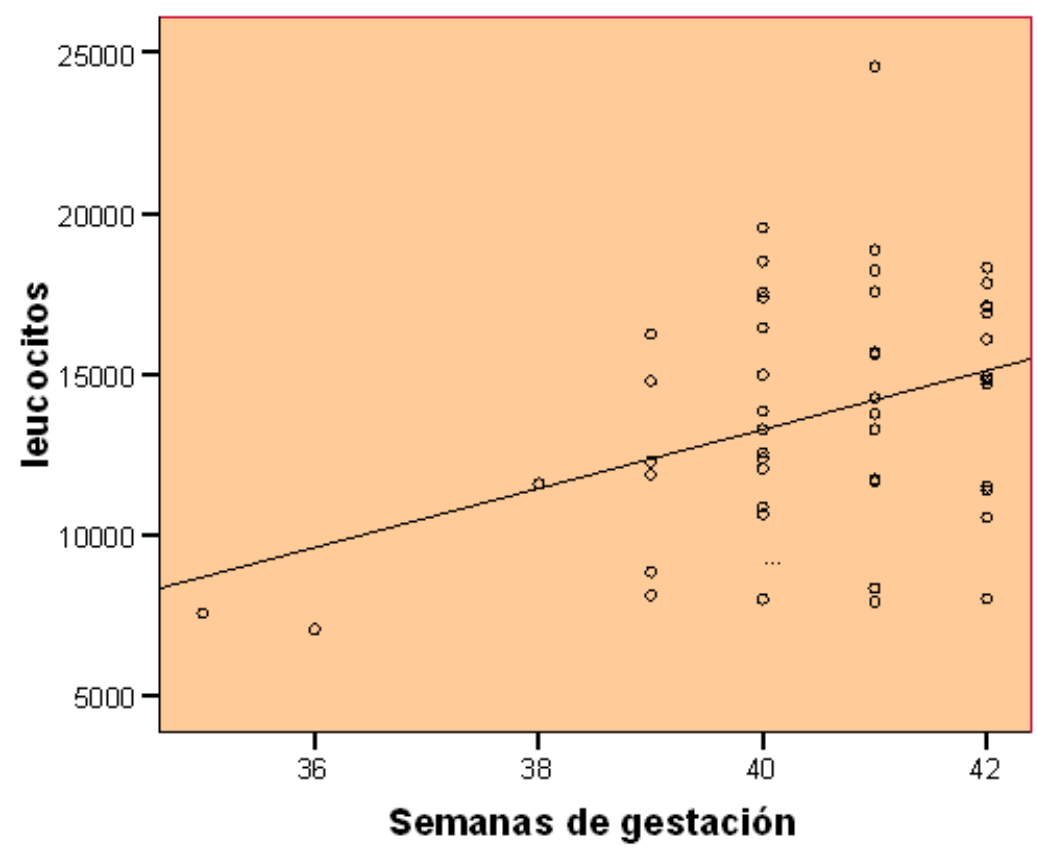


Figura 3: Correlación entre el peso del recien nacido y el peso inicial de la unidad de sangre de cordón en gramos.

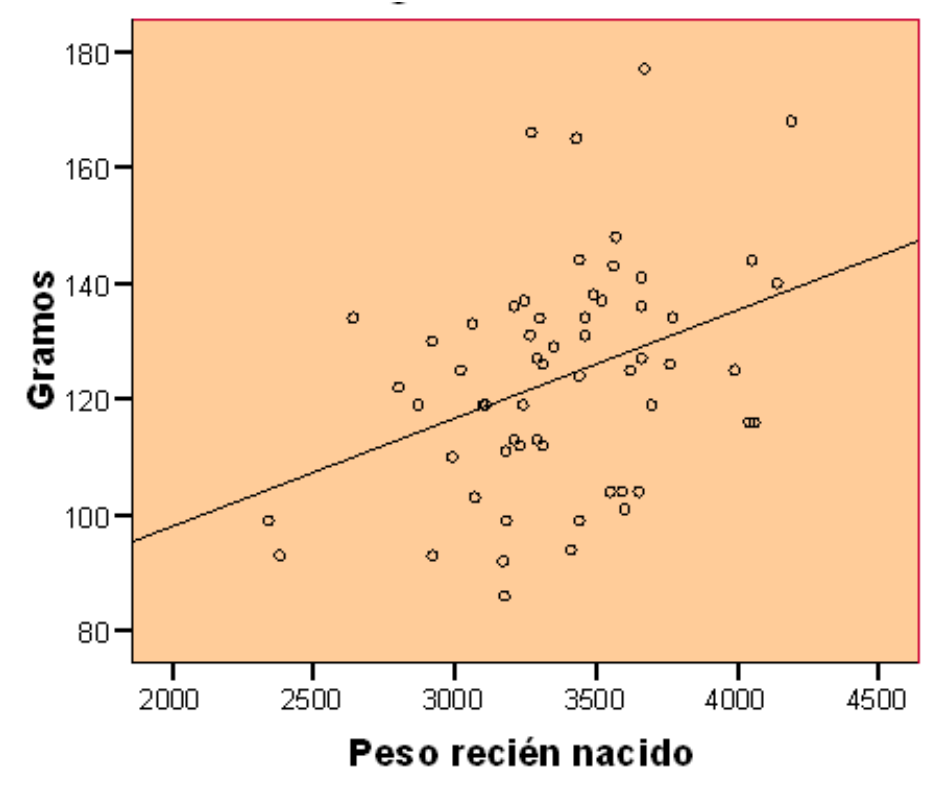

Figura 4: Peso incial de la unidad de SCU según paridad de la donante.

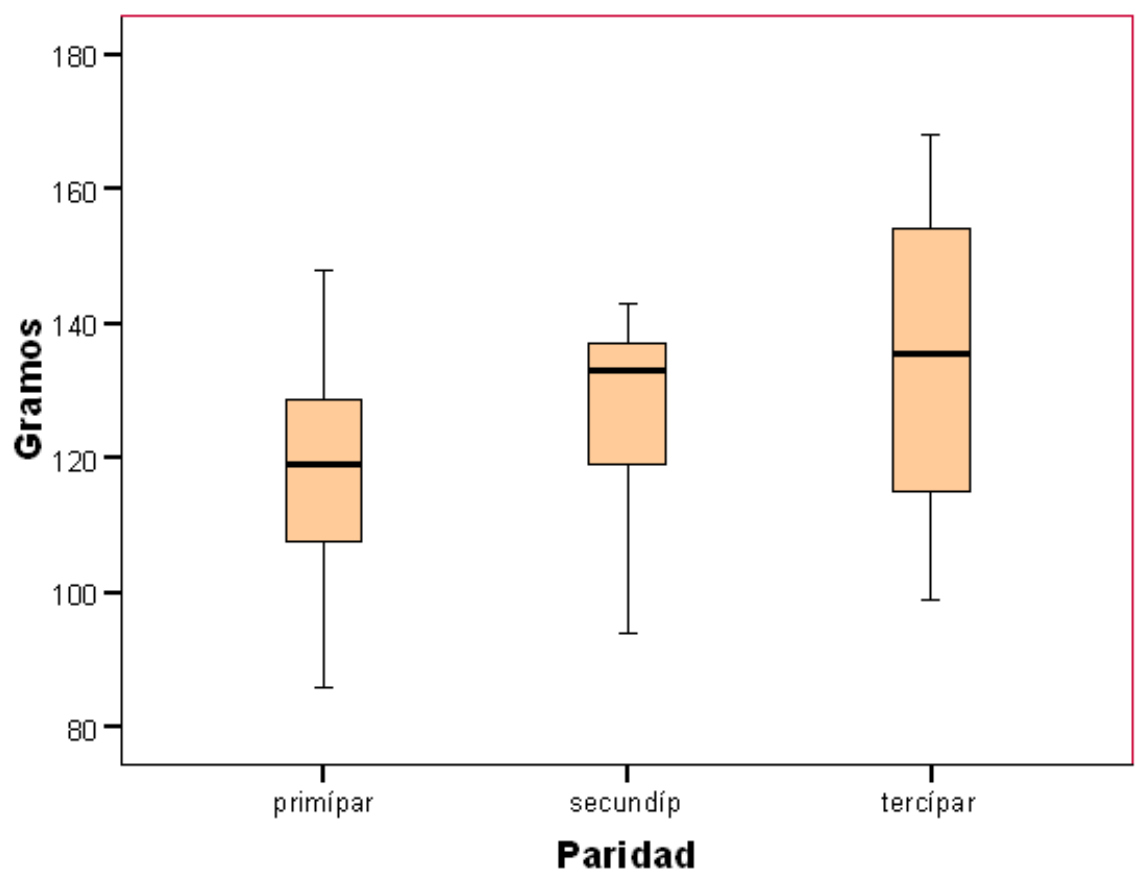

